\title{
Schwarzschild-Tangherlini black holes in five-dimensional massive (bi-)gravity
}

\author{
Tuan $Q . \mathrm{Do}^{1, *}$ \\ ${ }^{1}$ Faculty of Physics, VNU University of Science, Vietnam National University, Hanoi 120000, Vietnam
}

\begin{abstract}
We will present main results of our recent investigations on the existence of the Schwarzschild-Tangherlini black holes in a five-dimensional (nonlinear) massive gravity as well as in its dynamical extension, a fivedimensional massive bi-gravity. In particular, we will show how to use the well-known Cayley-Hamilton theorem to construct five- and higher dimensional massive graviton terms. Then, we will present the proof of the existence of the Schwarzschild-Tangherlini black holes in the five-dimensional massive (bi-)gravity.
\end{abstract}

\section{Introduction}

In 1939, Fierz and Pauli proposed a modification of the Einstein's general relativity (GR), in which the graviton is assumed to have a tiny but not vanishing mass $m_{g}$ [1]. However, this massive gravity theory was shown by van Dam-Veltman and Zakharov (vDVZ) in 1970 that it will not reduce to the GR in the limit $m_{g} \rightarrow 0[2,3]$. This problem has been well known as the vDVZ discontinuity. Two years later, Vainshtein proposed that nonlinear massive graviton terms might solve this discontinuity once they are introduced to the FP theory [4]. Soon after the publication of the Vainshtein's paper, however, Boulware and Deser (BD) showed that the nonlinear terms might introduce the existence of ghost mode, which is the sixth degree of freedom of the massive graviton [5]. As a result, this ghost mode was shown to has a negative kinetic energy, which causes an instability. Hence, it should be removed in order to ensure that the corresponding nonlinear massive gravity is stable. Unfortunately, building a nonlinear but ghost free massive gravity has been a great challenge to physicists for several decades [6]. From 2009 to 2010, based on some seminal works in Ref. [6], de Rham, Gabadadze, and Tolley (dRGT) proposed a version of nonlinear massive gravity [7], which was later shown to be free of the BD ghost by several different proofs [8]. Consequently, many physical and cosmological aspects have been studied in the context of this nonlinear massive gravity theory [9]. For example, the old cosmological constant problem and the dark energy problem might be solved in the context of the dRGT theory [9]. Furthermore, the Schwarzschild, Kerr, and charged black holes have been shown to exist in the dRGT theory, e.g., see Ref. [10]. In addition, a bi-gravity extension of the dRGT theory, in which a non-dynamical reference metric is introduced to be fully dynamical as a physical one, was proposed by Hassan and Rosen in Ref. [11]. Along with the dRGT theory, the bi-gravity has been discussed widely. Interested readers should read an interesting review [12] for more details.

\footnotetext{
*e-mail: tuanqdo@vnu.edu.vn
} 
It turns out that almost published papers on the (nonlinear) massive (bi-)gravity have worked only in four-dimensional (4D) spacetimes rather than higher dimensional ones $[9,12]$. Of course, there have been some papers on higher dimensional massive (bi-)gravity but they did not deal with any specific physical or cosmological solutions [13, 14]. In addition, some papers have studied higher dimensional black holes in the framework of the dRGT theory with only the first three massive graviton terms $\mathcal{L}_{2}, \mathcal{L}_{3}$, and $\mathcal{L}_{4}$ [15]. As we will show later, the contributions of higher dimensional graviton terms, e.g., $\mathcal{L}_{5}$, should not be ignored because these terms do exist in higher-than-four dimensional spacetimes, although they actually vanish in any 4D ones. Hence, we have proposed to study both higher dimensional nonlinear massive gravity and massive bi-gravity involving a full set of massive graviton terms in Refs. [16, 17]. Fortunately, we have found an effective way to construct arbitrary higher dimensional massive graviton terms for the dRGT theory by using the well-known CayleyHamilton theorem [18]. Then, we have chosen five-dimensional (5D) scenarios of massive (bi-)gravity with $\mathcal{L}_{5}$ as specific models to seek the well-known Schwarzschild-Tangherlini black holes [19].

This article is devoted to summarize some important results published in Refs. [16, 17]. It is organized as follows: (i) An introduction has been written in this section. (ii) Basic setup of the dRGT massive gravity and massive bi-gravity will be shown in Sec. 2. (iii) A construction of higher dimensional graviton terms will be presented in Sec. 3. (iv) Next, the Schwarzschild-Tangherlini black holes in the context of 5D massive (bi-)gravity will be shown to exist in Sec. 4. (v) Finally, concluding remarks will be given in Sec. 5.

\section{Basic setup of the dRGT massive gravity and massive bi-gravity}

In this section, we will present very briefly the basic setup of the dRGT massive gravity theory [7, 9] along with its important extension, the massive bi-gravity [11, 12]. As a result, an action of the dRGT massive gravity is given by [7]

$$
S_{\mathrm{dRGT}, 4 \mathrm{~d}}=\frac{M_{p}^{2}}{2} \int d^{4} x \sqrt{-g}\left\{R+m_{g}^{2}\left(\mathcal{L}_{2}+\alpha_{3} \mathcal{L}_{3}+\alpha_{4} \mathcal{L}_{4}\right)\right\},
$$

where $M_{p}$ is the reduced Planck mass, $m_{g} \neq 0$ is the graviton mass, and $\alpha_{3,4}$ are free parameters. In addition, $\mathcal{L}_{i}(i=2-4)$ are the massive graviton terms (a.k.a. interaction terms) defined as

$$
\begin{aligned}
& \mathcal{L}_{2}=[\mathcal{K}]^{2}-\left[\mathcal{K}^{2}\right], \\
& \mathcal{L}_{3}=\frac{1}{3}[\mathcal{K}]^{3}-[\mathcal{K}]\left[\mathcal{K}^{2}\right]+\frac{2}{3}\left[\mathcal{K}^{3}\right], \\
& \mathcal{L}_{4}=\frac{1}{12}[\mathcal{K}]^{4}-\frac{1}{2}[\mathcal{K}]^{2}\left[\mathcal{K}^{2}\right]+\frac{1}{4}\left[\mathcal{K}^{2}\right]^{2}+\frac{2}{3}[\mathcal{K}]\left[\mathcal{K}^{3}\right]-\frac{1}{2}\left[\mathcal{K}^{4}\right] .
\end{aligned}
$$

Here, square brackets stand for the trace of $\mathcal{K}^{\mu}{ }_{\nu} \equiv \delta^{\mu}{ }_{v}-\sqrt{g^{\mu \alpha} f_{a b} \partial_{\alpha} \phi^{a} \partial_{\nu} \phi^{b}}$, i.e.,

$$
[\mathcal{K}] \equiv \operatorname{tr} \mathcal{K}^{\mu}{ }_{\nu} ;[\mathcal{K}]^{n} \equiv\left(\operatorname{tr} \mathcal{K}^{\mu}{ }_{\nu}\right)^{n} ;\left[\mathcal{K}^{n}\right] \equiv \operatorname{tr}\left(\mathcal{K}^{\mu}{ }_{\alpha_{1}} \ldots \mathcal{K}^{\alpha_{n}}{ }_{\nu}\right) \text {. }
$$

Note that in the definition of $\mathcal{K}^{\mu}{ }_{\nu}, g_{\mu \nu}$ is regarded as the physical metric, while $f_{a b}$ the reference (or fiducial) metric, and $\phi^{a}(a=0-3)$ the Stückelberg scalar fields added to give a manifestly diffeomorphism invariant description $[6,7]$. It is noted that the reference metric in the dRGT massive gravity is non-dynamical, in contrast to the physical one, which is dynamical. Therefore, the field equations of $f_{a b}$ will turn out to be algebraic rather than differential. 
It is noted that if the reference metric is introduced to be fully dynamical as the physical one, we will have the massive bi-gravity (or bi-metric gravity), whose action is given by [11]

$S_{\text {Bigravity } 4 \mathrm{~d}}=M_{g}^{2} \int d^{4} x \sqrt{-g} R(g)+M_{f}^{2} \int d^{4} x \sqrt{-f} R(f)+2 m_{g}^{2} M_{\text {eff }}^{2} \int d^{4} x \sqrt{-g}\left(\mathcal{U}_{2}+\alpha_{3} \mathcal{U}_{3}+\alpha_{4} \mathcal{U}_{4}\right)$,

where $\mathcal{U}_{i}=\mathcal{L}_{i} / 2$, while $R(g)$ and $R(f)$ denote the corresponding Ricci scalar of the physical metric $g_{\mu \nu}$ and reference metric $f_{\mu \nu}$, respectively. It is noted that $M_{\text {eff }}$ is an effective Planck mass defined in terms of two different Planck masses of the physical and reference sectors $M_{g}$ and $M_{f}$, respectively, as follows [11]

$$
M_{\mathrm{eff}}^{2}=\left(\frac{1}{M_{g}^{2}}+\frac{1}{M_{f}^{2}}\right)^{-1}
$$

It is also noted that we do not need the existence of the Stückelberg scalar fields, i.e., $\mathcal{K}^{\mu}{ }_{v} \equiv$ $\delta^{\mu}{ }_{v}-\sqrt{g^{\mu \sigma} f_{\sigma v}}$ in the context of bi-gravity theory. As a consequence, the corresponding field equations of reference metric in the bi-gravity will no longer be algebraic but differential as that of physical metric. It is clear that $S_{\text {Bigravity,4d }} \rightarrow S_{\mathrm{dRGT}, 4 \mathrm{~d}}$ under the limit $M_{f} \rightarrow 0$. It turns out that both nonlinear massive gravity and massive bi-gravity theories have been shown to be free of the BD ghost via different approaches [8]. Furthermore, many cosmological aspects have been investigated in the framework of these theories, e.g., see Refs. [9, 12] for more details.

\section{Construction of higher dimensional graviton terms}

In mathematics, there exists the well-known Cayley-Hamilton theorem in linear algebra, e.g. see Ref. [18], whose statement is that any $n \times n$ matrix $K$ must obey its characteristic equation:

$$
\mathcal{P}(K) \equiv K^{n}-\mathcal{D}_{n-1} K^{n-1}+\mathcal{D}_{n-2} K^{n-2}-\ldots+(-1)^{n-1} \mathcal{D}_{1} K+(-1)^{n} \operatorname{det}(K) I_{n}=0,
$$

where $\mathcal{D}_{n-1}=\operatorname{tr} K \equiv[K]$ and $\mathcal{D}_{n-j}(2 \leq j \leq n-1)$ are coefficients of the characteristic polynomial. Surprisingly, it is straightforward to see that the forms of the first three graviton terms, $\mathcal{L}_{2}, \mathcal{L}_{3}$, and $\mathcal{L}_{4}$ look similar to the determinants of $K_{2 \times 2}, K_{3 \times 3}$, and $K_{4 \times 4}$, respectively. This is indeed a key observation to construct not only the 4D massive graviton terms but also any higher dimensional ones, i.e., $\mathcal{L}_{i>4}[12-14,16,17]$. As a result, it turns out that $\mathcal{L}_{i}=2 \operatorname{det} \mathcal{K}_{i \times i}$ with $i=2-n$. For example, we have constructed five-, six-, and sevendimensional graviton terms as specific demonstrations $[16,17]$ :

$$
\begin{aligned}
\frac{\mathcal{L}_{5}}{2}= & \frac{1}{120}\left\{[\mathcal{K}]^{5}-10[\mathcal{K}]^{3}\left[\mathcal{K}^{2}\right]+20[\mathcal{K}]^{2}\left[\mathcal{K}^{3}\right]-20\left[\mathcal{K}^{2}\right]\left[\mathcal{K}^{3}\right]+15[\mathcal{K}]\left[\mathcal{K}^{2}\right]^{2}\right. \\
& \left.-30[\mathcal{K}]\left[\mathcal{K}^{4}\right]+24\left[\mathcal{K}^{5}\right]\right\} \\
\frac{\mathcal{L}_{6}}{2}= & \frac{1}{720}\left\{[\mathcal{K}]^{6}-15[\mathcal{K}]^{4}\left[\mathcal{K}^{2}\right]+40[\mathcal{K}]^{3}\left[\mathcal{K}^{3}\right]-90[\mathcal{K}]^{2}\left[\mathcal{K}^{4}\right]+45[\mathcal{K}]^{2}\left[\mathcal{K}^{2}\right]^{2}-15\left[\mathcal{K}^{2}\right]^{3}\right. \\
& \left.+40\left[\mathcal{K}^{3}\right]^{2}-120\left[\mathcal{K}^{3}\right]\left[\mathcal{K}^{2}\right][\mathcal{K}]+90\left[\mathcal{K}^{4}\right]\left[\mathcal{K}^{2}\right]+144\left[\mathcal{K}^{5}\right][\mathcal{K}]-120\left[\mathcal{K}^{6}\right]\right\} \\
\frac{\mathcal{L}_{7}}{2}= & \frac{1}{5040}\left\{[\mathcal{K}]^{7}-21[\mathcal{K}]^{5}\left[\mathcal{K}^{2}\right]+70[\mathcal{K}]^{4}\left[\mathcal{K}^{3}\right]-210[\mathcal{K}]^{3}\left[\mathcal{K}^{4}\right]+105[\mathcal{K}]^{3}\left[\mathcal{K}^{2}\right]^{2}\right. \\
& -420[\mathcal{K}]^{2}\left[\mathcal{K}^{2}\right]\left[\mathcal{K}^{3}\right]+504[\mathcal{K}]^{2}\left[\mathcal{K}^{5}\right]-105\left[\mathcal{K}^{2}\right]^{3}[\mathcal{K}]+210\left[\mathcal{K}^{2}\right]^{2}\left[\mathcal{K}^{3}\right]-504\left[\mathcal{K}^{2}\right]\left[\mathcal{K}^{5}\right] \\
& \left.+280\left[\mathcal{K}^{3}\right]^{2}[\mathcal{K}]-420\left[\mathcal{K}^{3}\right]\left[\mathcal{K}^{4}\right]+630\left[\mathcal{K}^{4}\right]\left[\mathcal{K}^{2}\right][\mathcal{K}]-840\left[\mathcal{K}^{6}\right][\mathcal{K}]+720\left[\mathcal{K}^{7}\right]\right\}
\end{aligned}
$$


Note that these terms all disappear identically in any lower-than-five dimensional spacetimes. However, $\mathcal{L}_{n}$ would not vanish in any spacetimes, whose number of dimensions is greater than or equal to $n$. This fact can be easily verified using the Cayley-Hamilton theorem. Unfortunately, some papers investigating black holes solutions of higher dimensional dRGT gravity have ignored this important result [15].

\section{Schwarzschild-Tangherlini black holes}

In this section, we will show how the Schwarzschild-Tangherlini black holes [19] do appear in both 5D massive gravity [16] and bi-gravity [17].

\subsection{D massive gravity}

First, we would like to investigate the 5D dRGT gravity with the following action [16],

$$
S_{\mathrm{dRGT}, 5 \mathrm{~d}}=\frac{M_{p}^{2}}{2} \int d^{5} x \sqrt{-g}\left\{R+m_{g}^{2}\left(\mathcal{L}_{2}+\alpha_{3} \mathcal{L}_{3}+\alpha_{4} \mathcal{L}_{4}+\alpha_{5} \mathcal{L}_{5}\right)\right\},
$$

here $\alpha_{5}$ is an additional field parameter introduced for the additional massive graviton term $\mathcal{L}_{5}$ defined in Eq. (9) with $K=\mathcal{K}$. As a result, varying the action (12) w.r.t. the inverse metric $g^{\mu \nu}$ leads to the corresponding Einstein field equations for the physical metric $[13,14,16]$

$$
\left(R_{\mu \nu}-\frac{1}{2} R g_{\mu \nu}\right)+m_{g}^{2}\left(X_{\mu \nu}+\sigma Y_{\mu \nu}+\alpha_{5} W_{\mu \nu}\right)=0
$$

where

$$
\begin{aligned}
X_{\mu \nu} & =-\frac{1}{2}\left(\alpha \mathcal{L}_{2}+\beta \mathcal{L}_{3}\right) g_{\mu \nu}+\tilde{X}_{\mu \nu} \\
\tilde{X}_{\mu \nu} & =\mathcal{K}_{\mu \nu}-[\mathcal{K}] g_{\mu \nu}-\alpha\left\{\mathcal{K}_{\mu \nu}^{2}-[\mathcal{K}] \mathcal{K}_{\mu \nu}\right\}+\beta\left\{\mathcal{K}_{\mu \nu}^{3}-[\mathcal{K}] \mathcal{K}_{\mu \nu}^{2}+\frac{\mathcal{L}_{2}}{2} \mathcal{K}_{\mu \nu}\right\} \\
Y_{\mu \nu} & =-\frac{\mathcal{L}_{4}}{2} g_{\mu \nu}+\tilde{Y}_{\mu \nu} ; \tilde{Y}_{\mu \nu}=\frac{\mathcal{L}_{3}}{2} \mathcal{K}_{\mu \nu}-\frac{\mathcal{L}_{2}}{2} \mathcal{K}_{\mu \nu}^{2}+[\mathcal{K}] \mathcal{K}_{\mu \nu}^{3}-\mathcal{K}_{\mu \nu}^{4} \\
W_{\mu \nu} & =-\frac{\mathcal{L}_{5}}{2} g_{\mu \nu}+\tilde{W}_{\mu \nu} ; \tilde{W}_{\mu \nu}=\frac{\mathcal{L}_{4}}{2} \mathcal{K}_{\mu \nu}-\frac{\mathcal{L}_{3}}{2} \mathcal{K}_{\mu \nu}^{2}+\frac{\mathcal{L}_{2}}{2} \mathcal{K}_{\mu \nu}^{3}-[\mathcal{K}] \mathcal{K}_{\mu \nu}^{4}+\mathcal{K}_{\mu \nu}^{5},
\end{aligned}
$$

with $\alpha \equiv 1+\alpha_{3}, \beta \equiv \alpha_{3}+\alpha_{4}$, and $\sigma \equiv \alpha_{4}+\alpha_{5}$ being additional parameters introduced for convenience. On the other hand, the corresponding constraint equations associated with the existence of reference metric turn out to be

$$
t_{\mu \nu} \equiv \tilde{X}_{\mu \nu}+\sigma \tilde{Y}_{\mu \nu}+\alpha_{5} \tilde{W}_{\mu \nu}-\frac{1}{2}\left(\alpha_{3} \mathcal{L}_{2}+\alpha_{4} \mathcal{L}_{3}+\alpha_{5} \mathcal{L}_{4}\right) g_{\mu \nu}=0 .
$$

Furthermore, the modified Einstein field equations (13) can be reduced to [20]

$$
\left(R_{\mu \nu}-\frac{1}{2} R g_{\mu \nu}\right)-\frac{m_{g}^{2}}{2} \mathcal{L}_{M} g_{\mu \nu}=0,
$$

with $\mathcal{L}_{M} \equiv \mathcal{L}_{2}+\alpha_{3} \mathcal{L}_{3}+\alpha_{4} \mathcal{L}_{4}+\alpha_{5} \mathcal{L}_{5}$ as the total massive graviton Lagrangian. The equation (19) shows an interesting result that the massive graviton terms can play as an effective cosmological constant, i.e., $\Lambda_{M} \equiv-m_{g}^{2} \mathcal{L}_{M} / 2$, as a consequence of the Bianchi identity, i.e., $\partial^{v} \mathcal{L}_{M}=0$. Indeed, this result has been shown to be valid for a large class of physical and 
compatible reference metrics [16]. It turns out that the corresponding values of $\Lambda_{M}$ could be figured out once the constraint equations (18), which are indeed algebraic rather than differential, are solved [16].

In order to seek the Schwarzschild-Tangherlini metric for the 5D dRGT gravity, let us consider the general forms for both physical and reference metrics [16],

$$
d s_{5 d}^{2}(g)=-N_{1}^{2}(r) d t^{2}+\frac{d r^{2}}{F_{1}^{2}(r)}+\frac{r^{2} d \Omega_{3}^{2}}{H_{1}^{2}(r)} ; d s_{5 d}^{2}(f)=-N_{2}^{2}(r) d t^{2}+\frac{d r^{2}}{F_{2}^{2}(r)}+\frac{r^{2} d \Omega_{3}^{2}}{H_{2}^{2}(r)},
$$

with $d \Omega_{3}^{2}=d \theta^{2}+\sin ^{2} \theta d \varphi^{2}+\sin ^{2} \theta \sin ^{2} \varphi d \psi^{2}$. Here, $N_{1}(r)$ and $N_{2}(r)$ are the lapse functions, which are introduced to obtain the following Friedmann equations from their Euler-Lagrange equations [20]. Consequently, the corresponding $\mathcal{L}_{M}$ now reads

$$
\begin{aligned}
\mathcal{L}_{M} & =2\left\{\left[\alpha_{5}\left(\mathcal{K}^{2}{ }_{2}\right)^{3}+3 \alpha_{4}\left(\mathcal{K}^{2}{ }_{2}\right)^{2}+3 \alpha_{3} \mathcal{K}^{2}{ }_{2}+1\right] \mathcal{K}_{0}^{0} \mathcal{K}^{1}{ }_{1}\right. \\
& \left.+\mathcal{K}^{2}{ }_{2}\left[\alpha_{4}\left(\mathcal{K}^{2}{ }_{2}\right)^{2}+3 \alpha_{3} \mathcal{K}^{2}{ }_{2}+3\right]\left(\mathcal{K}_{0}^{0}+\mathcal{K}^{1}{ }_{1}\right)+\left(\mathcal{K}^{2}{ }_{2}\right)^{2}\left(\alpha_{3} \mathcal{K}^{2}{ }_{2}+3\right)\right\},
\end{aligned}
$$

where $\mathcal{K}^{0}{ }_{0}(r)=1-N_{2} / N_{1} ; \mathcal{K}^{1}{ }_{1}(r)=1-F_{1} / F_{2} ; \mathcal{K}^{2}{ }_{2}(r)=\mathcal{K}^{3}{ }_{3}(r)=\mathcal{K}^{4}{ }_{4}(r)=1-H_{1} / H_{2}$. Note that the unitary gauge for the Stückelberg scalar fields, i.e., $\phi^{a}=x^{a}(a=0-4)$, has been used in order to derive the above results of $\mathcal{K}^{\mu}{ }_{v}$ and $\mathcal{L}_{M}$. Next, we derive explicitly the following constraint equations for the 5D dRGT gravity $t_{\mu v}=0$. As a result, these equations are indeed equivalent to the corresponding Euler-Lagrange equations $\partial \mathcal{L}_{M} / \partial \mathcal{K}^{0}{ }_{0}=$ $\partial \mathcal{L}_{M} / \partial \mathcal{K}^{1}{ }_{1}=\partial \mathcal{L}_{M} / \partial \mathcal{K}^{2}{ }_{2}=0$, which can be easily defined to be

$$
\begin{aligned}
& \left(\alpha_{5} \mathcal{K}^{1}{ }_{1}+\alpha_{4}\right)\left(\mathcal{K}^{2}{ }_{2}\right)^{3}+3\left(\alpha_{4} \mathcal{K}^{1}{ }_{1}+\alpha_{3}\right)\left(\mathcal{K}^{2}\right)^{2}+3\left(\alpha_{3} \mathcal{K}^{1}{ }_{1}+1\right) \mathcal{K}^{2}{ }_{2}+\mathcal{K}^{1}{ }_{1}=0, \\
& \left(\alpha_{5} \mathcal{K}_{0}^{0}{ }_{0}+\alpha_{4}\right)\left(\mathcal{K}^{2}{ }_{2}\right)^{3}+3\left(\alpha_{4} \mathcal{K}^{0}{ }_{0}+\alpha_{3}\right)\left(\mathcal{K}^{2}{ }_{2}\right)^{2}+3\left(\alpha_{3} \mathcal{K}^{0}{ }_{0}+1\right) \mathcal{K}^{2}{ }_{2}+\mathcal{K}^{0}{ }_{0}=0 \text {, } \\
& {\left[\alpha_{5}\left(\mathcal{K}^{2}{ }_{2}\right)^{2}+2 \alpha_{4} \mathcal{K}^{2}{ }_{2}+\alpha_{3}\right] \mathcal{K}^{0}{ }_{0} \mathcal{K}^{1}{ }_{1}+\left[\alpha_{4}\left(\mathcal{K}^{2}{ }_{2}\right)^{2}+2 \alpha_{3} \mathcal{K}^{2}{ }_{2}+1\right]\left(\mathcal{K}^{0}{ }_{0}+\mathcal{K}^{1}{ }_{1}\right)} \\
& +\left(\alpha_{3} \mathcal{K}_{2}^{2}+2\right) \mathcal{K}_{2}^{2}=0
\end{aligned}
$$

respectively. As a result, combining two Eqs. (22) and (23) leads to

$$
\left[\alpha_{5}\left(\mathcal{K}_{2}^{2}\right)^{3}+3 \alpha_{4}\left(\mathcal{K}_{2}^{2}\right)^{2}+3 \alpha_{3} \mathcal{K}_{2}^{2}+1\right]\left(\mathcal{K}_{0}^{0}-\mathcal{K}^{1}{ }_{1}\right)=0
$$

which implies two possible cases:

$$
\begin{aligned}
\mathcal{K}^{0}{ }_{0} & =\mathcal{K}^{1}{ }_{1}, \\
\alpha_{5}\left(\mathcal{K}^{2}{ }_{2}\right)^{3}+3 \alpha_{4}\left(\mathcal{K}^{2}{ }_{2}\right)^{2}+3 \alpha_{3} \mathcal{K}^{2}{ }_{2}+1 & =0 .
\end{aligned}
$$

As a result, these equations are non-linear of $\mathcal{K}^{0}{ }_{0}, \mathcal{K}^{1}{ }_{1}$, and $\mathcal{K}^{2}{ }_{2}$. Therefore, solving them will imply several values of $\mathcal{L}_{M}$.

Case 1: $\mathcal{K}_{0}^{0}=\mathcal{K}^{1}{ }_{1}$.

For this case, we have been able to obtain two solutions such as

$$
\begin{aligned}
& \mathcal{K}^{1}{ }_{1}=\mathcal{K}^{2}{ }_{2}, \\
& \mathcal{K}^{1}{ }_{1}=-\frac{\alpha_{4}\left(\mathcal{K}^{2}{ }_{2}\right)^{2}+2 \alpha_{3} \mathcal{K}^{2}{ }_{2}+1}{\alpha_{5}\left(\mathcal{K}^{2}{ }_{2}\right)^{2}+2 \alpha_{4} \mathcal{K}^{2}{ }_{2}+\alpha_{3}} .
\end{aligned}
$$


For the first solution (28), we arrive at the following equation for $\mathcal{K}^{2}$,

$$
\alpha_{5}\left(\mathcal{K}_{2}^{2}\right)^{3}+4 \alpha_{4}\left(\mathcal{K}_{2}^{2}\right)^{2}+6 \alpha_{3} \mathcal{K}_{2}^{2}+4=0
$$

along with the value of an effective cosmological constant derived from $\mathcal{L}_{M}$,

$$
\Lambda_{M} \equiv-\frac{m_{g}^{2} \mathcal{L}_{M}}{2}=-m_{g}^{2}\left(\mathcal{K}_{2}^{2}\right)^{2}\left[\alpha_{4}\left(\mathcal{K}_{2}^{2}\right)^{2}+4 \alpha_{3} \mathcal{K}^{2}{ }_{2}+6\right]
$$

It is clear that once Eq. (30) is solved, the value of $\Lambda_{M}$ will be obtained accordingly.

For the second solution (29), the following equation for $\mathcal{K}^{2}{ }_{2}$ reads

$$
\left(\alpha_{3} \alpha_{5}-\alpha_{4}^{2}\right)\left(\mathcal{K}_{2}^{2}\right)^{4}+2\left(\alpha_{5}-\alpha_{3} \alpha_{4}\right)\left(\mathcal{K}_{2}^{2}\right)^{3}+\left(2 \alpha_{4}-3 \alpha_{3}^{2}\right)\left(\mathcal{K}_{2}^{2}\right)^{2}-2 \alpha_{3} \mathcal{K}_{2}^{2}-1=0
$$

And of course, once $\mathcal{K}^{2}{ }_{2}$ is solved, the corresponding $\mathcal{K}^{0}{ }_{0}$ and $\mathcal{K}^{1}{ }_{1}$ and therefore $\Lambda_{M}$ will be determined consistently.

Case 2: $\alpha_{5}\left(\mathcal{K}_{2}^{2}\right)^{3}+3 \alpha_{4}\left(\mathcal{K}_{2}^{2}\right)^{2}+3 \alpha_{3} \mathcal{K}_{2}^{2}+1=0$.

As a result, we have figured out the corresponding results such as

$$
\begin{aligned}
& \mathcal{K}_{2}^{2}=\frac{-3 \alpha_{3} \pm \sqrt{9 \alpha_{3}^{2}-12 \alpha_{4}}}{2 \alpha_{4}} ; \alpha_{5}=\frac{8 \alpha_{4}^{2}\left[\left(9 \alpha_{3}^{2}-8 \alpha_{4}\right) \mp 3 \alpha_{3} \sqrt{9 \alpha_{3}^{2}-12 \alpha_{4}}\right]}{\left(3 \alpha_{3} \mp \sqrt{9 \alpha_{3}^{2}-12 \alpha_{4}}\right)^{3}}, \\
& \Lambda_{M}=\frac{3 m_{g}^{2}}{2 \alpha_{4}^{3}}\left[9 \alpha_{3}^{4}-18 \alpha_{3}^{2} \alpha_{4}+6 \alpha_{4}^{2} \mp \alpha_{3}\left(3 \alpha_{3}^{2}-4 \alpha_{4}\right) \sqrt{9 \alpha_{3}^{2}-12 \alpha_{4}}\right] .
\end{aligned}
$$

It appears that the value $\Lambda_{M}$ has been obtained without knowing that of $\mathcal{K}^{0}{ }_{0}$ and $\mathcal{K}^{1}{ }_{1}$, which can be found from the remaining constraint equation

$$
\left(\alpha_{3} \mathcal{K}_{2}^{2}+2\right)\left[\mathcal{K}_{2}^{2}\left(\mathcal{K}^{2}{ }_{2}-\mathcal{K}_{0}^{0}-\mathcal{K}^{1}{ }_{1}\right)+\mathcal{K}_{0}^{0}{ }_{0} \mathcal{K}_{1}^{1}\right]=0
$$

As a result, solving this equation implies two solutions, one is that

$$
\mathcal{K}_{0}^{0}=\mathcal{K}_{1}^{1}=\mathcal{K}_{2}^{2}=\frac{-3 \alpha_{3} \pm \sqrt{9 \alpha_{3}^{2}-12 \alpha_{4}}}{2 \alpha_{4}},
$$

while the other is given by

$$
\mathcal{K}_{2}^{2}=-\frac{2}{\alpha_{3}} ; \alpha_{4}=\frac{3 \alpha_{3}^{2}}{4} ; \alpha_{5}=\frac{\alpha_{3}^{3}}{2},
$$

which implies that

$$
\Lambda_{M}=-\frac{4 m_{g}^{2}}{\alpha_{3}^{2}}<0 ; \mathcal{K}^{0}{ }_{0}=\mathcal{K}^{1}{ }_{1}=\mathcal{K}^{2}{ }_{2}=-\frac{2}{\alpha_{3}} .
$$

In short, the massive graviton term $\mathcal{L}_{M}$ always acts as an effective cosmological constant $\Lambda_{M}$. Hence, the 5D Einstein field equations (19) can be reduced to the following simple form as

$$
\left(R_{\mu \nu}-\frac{1}{2} R g_{\mu \nu}\right)+\Lambda_{M} g_{\mu \nu}=0
$$


here $\Lambda_{M} \equiv-m_{q}^{2} \mathcal{L}_{M} / 2$ can be either negative or positive or even zero definite depending on the values of field parameters $\alpha_{i}(i=3-5)$. It has been shown that in Ref. [19] the 5D Einstein equations with a cosmological constant $\Lambda_{M}$ as shown in Eq. (39) do admit the following metric,

$$
d s^{2}=-f(r) d t^{2}+\frac{d r^{2}}{f(r)}+r^{2} d \Omega_{3}^{2},
$$

with

$$
N_{1}^{2}(t, r)=F_{1}^{2}(t, r)=f(r)=1-\frac{\mu}{r^{2}}-\frac{\Lambda_{M}}{6} r^{2} ; H_{1}^{2}(t, r)=1 ; \mu=\frac{8 G_{5} M}{3 \pi},
$$

as their solutions [19]. Here $M$ and $G_{5}$ stand for the mass of source and the 5D Newton constant, respectively. As a result, this solution is called the Schwarzschild-Tangherlini-de Sitter black holes if $\Lambda_{M}>0$, the Schwarzschild-Tangherlini-anti-de Sitter black holes if $\Lambda_{M}<0$, and the Schwarzschild-Tangherlini black holes if $\Lambda_{M}=0$ [19].

\subsection{D massive bi-gravity}

The corresponding action of 5D massive bi-gravity has been given by [17]

$$
\begin{aligned}
S_{\text {Bigravity }, 5 \mathrm{~d}} & =M_{g}^{2} \int d^{5} x \sqrt{-g} R(g)+M_{f}^{2} \int d^{5} x \sqrt{-f} R(f) \\
& +2 m_{g}^{2} M_{\mathrm{eff}}^{2} \int d^{5} x \sqrt{-g}\left(\mathcal{U}_{2}+\alpha_{3} \mathcal{U}_{3}+\alpha_{4} \mathcal{U}_{4}+\alpha_{5} \mathcal{U}_{5}\right),
\end{aligned}
$$

with $\mathcal{U}_{i}=\mathcal{L}_{i} / 2(i=2-5)$ and therefore $\mathcal{U}_{M} \equiv \mathcal{U}_{2}+\alpha_{3} \mathcal{U}_{3}+\alpha_{4} \mathcal{U}_{4}+\alpha_{5} \mathcal{U}_{5}=\mathcal{L}_{M} / 2$. As a result, the corresponding 5D Einstein field equations turn out to be [13, 14, 17]

$$
M_{g}^{2}\left(R_{\mu \nu}(g)-\frac{1}{2} g_{\mu \nu} R(g)\right)+m_{g}^{2} M_{\text {eff }}^{2} \mathcal{H}_{\mu \nu}^{(5)}(g)=0,
$$

where $\mathcal{H}_{\mu \nu}^{(5)}(g) \equiv X_{\mu \nu}+\sigma Y_{\mu \nu}+\alpha_{5} W_{\mu \nu}$. In addition, the tensors $X_{\mu \nu}, Y_{\mu \nu}$, and $W_{\mu \nu}$ have been defined in Eqs. (14), (16), and (17), respectively. It is clear that Eq. (43) will become identical to Eq. (13) if $M_{f}=0$. Now, for the reference metric, we can derive its field equations to be

$$
\sqrt{-f} M_{f}^{2}\left(R_{\mu \nu}(f)-\frac{1}{2} f_{\mu \nu} R(f)\right)+\sqrt{-g} m_{g}^{2} M_{\mathrm{eff}}^{2} s_{\mu \nu}^{(5)}(f)=0
$$

where the tensor $s_{\mu \nu}^{(5)}(f)$ is given by

$$
\begin{aligned}
s_{\mu \nu}^{(5)}(f) & \equiv-\hat{\mathcal{K}}_{\mu \nu}+\left\{[\mathcal{K}]+\alpha_{3} \mathcal{U}_{2}+\alpha_{4} \mathcal{U}_{3}+\alpha_{5} \mathcal{U}_{4}\right\} f_{\mu \nu}+\alpha\left\{\hat{\mathcal{K}}_{\mu \nu}^{2}-[\mathcal{K}] \hat{\mathcal{K}}_{\mu \nu}\right\} \\
& -\beta\left\{\hat{\mathcal{K}}_{\mu \nu}^{3}-[\mathcal{K}] \hat{\mathcal{K}}_{\mu \nu}^{2}+\mathcal{U}_{2} \hat{\mathcal{K}}_{\mu \nu}\right\}-\sigma\left\{\mathcal{U}_{3} \hat{\mathcal{K}}_{\mu \nu}-\mathcal{U}_{2} \hat{\mathcal{K}}_{\mu \nu}^{2}+[\mathcal{K}] \hat{\mathcal{K}}_{\mu \nu}^{3}-\hat{\mathcal{K}}_{\mu \nu}^{4}\right\} \\
& -\alpha_{5}\left\{\mathcal{U}_{4} \hat{\mathcal{K}}_{\mu \nu}-\mathcal{U}_{3} \hat{\mathcal{K}}_{\mu \nu}^{2}+\mathcal{U}_{2} \hat{\mathcal{K}}_{\mu \nu}^{3}-[\mathcal{K}] \hat{\mathcal{K}}_{\mu \nu}^{4}+\hat{\mathcal{K}}_{\mu \nu}^{5}\right\} .
\end{aligned}
$$

where the hatted tensors are defined as $\hat{\mathcal{K}}_{\mu v}=\mathcal{K}_{\mu}^{\sigma} f_{\sigma v}, \ldots, \hat{\mathcal{K}}_{\mu \nu}^{5}=\mathcal{K}_{\mu}^{\rho} \mathcal{K}_{\rho}^{\delta} \mathcal{K}_{\delta}^{\gamma} \mathcal{K}_{\gamma}^{\sigma} \mathcal{K}_{\sigma}^{\alpha} f_{\alpha v}$ for convenience. It is clear that Eq. (44) will no longer be algebraic but differential. Hence, it is not easy to have the constant-like behavior of graviton terms in the context of bi-gravity. Note, however, that there are two constraint equations due to the Bianchi identities for the physical and reference metrics in the 5D bi-gravity $[13,14,17]$

$$
D_{g}^{\mu} \mathcal{H}_{\mu \nu}^{(5)}(g)=0 ; D_{f}^{\mu}\left[\frac{\sqrt{-g}}{\sqrt{-f}} s_{\mu \nu}^{(5)}(f)\right]=0
$$


where we use notations $D_{g}^{\mu}$ and $D_{f}^{\mu}$ for the covariant derivatives in the physical and reference sectors, respectively. Given the metrics shown in Eqs. (20), we have been able to define explicitly components of Eq. (46) to be

$$
\begin{aligned}
& g^{11}\left[\partial_{r} \mathcal{H}_{11}^{(5)}-2 \Gamma_{11}^{1}(g) \mathcal{H}_{11}^{(5)}\right]-g^{00}\left[\Gamma_{00}^{1}(g) \mathcal{H}_{11}^{(5)}+\Gamma_{01}^{0}(g) \mathcal{H}_{00}^{(5)}\right] \\
& -g^{22}\left[\Gamma_{22}^{1}(g) \mathcal{H}_{11}^{(5)}+\Gamma_{21}^{2}(g) \mathcal{H}_{22}^{(5)}\right]-g^{33}\left[\Gamma_{33}^{1}(g) \mathcal{H}_{11}^{(5)}+\Gamma_{31}^{3}(g) \mathcal{H}_{33}^{(5)}\right] \\
& -g^{44}\left[\Gamma_{44}^{1}(g) \mathcal{H}_{11}^{(5)}+\Gamma_{41}^{4}(g) \mathcal{H}_{44}^{(5)}\right]=0, \\
& g^{33}\left[\Gamma_{33}^{2}(g) \mathcal{H}_{22}^{(5)}+\Gamma_{32}^{3}(g) \mathcal{H}_{33}^{(5)}\right]+g^{44}\left[\Gamma_{44}^{2}(g) \mathcal{H}_{22}^{(5)}+\Gamma_{42}^{4}(g) \mathcal{H}_{44}^{(5)}\right]=0, \\
& g^{44}\left[\Gamma_{44}^{3}(g) \mathcal{H}_{33}^{(5)}+\Gamma_{43}^{4}(g) \mathcal{H}_{44}^{(5)}\right]=0, \\
& f^{11}\left\{\partial_{r}\left[\frac{\sqrt{-g}}{\sqrt{-f}} s_{11}^{(5)}\right]-2 \frac{\sqrt{-g}}{\sqrt{-f}} \Gamma_{11}^{1}(f) s_{11}^{(5)}\right\}-\frac{\sqrt{-g}}{\sqrt{-f}} f^{00}\left[\Gamma_{00}^{1}(f) s_{11}^{(5)}+\Gamma_{01}^{0}(f) s_{00}^{(5)}\right] \\
& -\frac{\sqrt{-g}}{\sqrt{-f}} f^{22}\left[\Gamma_{22}^{1}(f) s_{11}^{(5)}+\Gamma_{21}^{2}(f) s_{22}^{(5)}\right]-\frac{\sqrt{-g}}{\sqrt{-f}} f^{33}\left[\Gamma_{33}^{1}(f) s_{11}^{(5)}+\Gamma_{31}^{3}(f) s_{33}^{(5)}\right] \\
& -\frac{\sqrt{-g}}{\sqrt{-f}} f^{44}\left[\Gamma_{44}^{1}(f) s_{11}^{(5)}+\Gamma_{41}^{4}(f) s_{44}^{(5)}\right]=0, \\
& f^{33}\left[\Gamma_{33}^{2}(f) s_{22}^{(5)}+\Gamma_{32}^{3}(f) s_{33}^{(5)}\right]+f^{44}\left[\Gamma_{44}^{2}(f) s_{22}^{(5)}+\Gamma_{42}^{4}(f) s_{44}^{(5)}\right]=0, \\
& f^{44}\left[\Gamma_{44}^{3}(f) s_{33}^{(5)}+\Gamma_{43}^{4}(f) s_{44}^{(5)}\right]=0 .
\end{aligned}
$$

Note that the following definition of $\mathcal{H}_{\mu \nu}^{(5)}(g)$ is given by

$$
\begin{aligned}
\mathcal{H}_{00}^{(5)} & =-\left[\sigma \mathcal{K}^{1}{ }_{1}\left(\mathcal{K}^{2}{ }_{2}\right)^{3}+\beta\left(\mathcal{K}^{2}{ }_{2}\right)^{2}\left(3 \mathcal{K}^{1}{ }_{1}+\mathcal{K}^{2}{ }_{2}\right)+3 \alpha \mathcal{K}^{2}{ }_{2}\left(\mathcal{K}^{1}{ }_{1}+\mathcal{K}^{2}{ }_{2}\right)+\mathcal{K}^{1}{ }_{1}+3 \mathcal{K}^{2}{ }_{2}\right] g_{00}, \\
\mathcal{H}_{11}^{(5)} & =-\left[\sigma \mathcal{K}^{0}{ }_{0}\left(\mathcal{K}^{2}{ }_{2}\right)^{3}+\beta\left(\mathcal{K}^{2}{ }_{2}\right)^{2}\left(3 \mathcal{K}^{0}{ }_{0}+\mathcal{K}^{2}{ }_{2}\right)+3 \alpha \mathcal{K}^{2}{ }_{2}\left(\mathcal{K}^{0}{ }_{0}+\mathcal{K}^{2}{ }_{2}\right)+\mathcal{K}^{0}{ }_{0}+3 \mathcal{K}^{2}{ }_{2}\right] g_{11}, \\
\mathcal{H}_{22}^{(5)} & =-\left\{\sigma \mathcal{K}_{0}^{0} \mathcal{K}^{1}{ }_{1}\left(\mathcal{K}^{2}{ }_{2}\right)^{2}+\beta \mathcal{K}^{2}{ }_{2}\left(2 \mathcal{K}^{0}{ }_{0} \mathcal{K}^{1}{ }_{1}+\mathcal{K}_{0}{ }_{0} \mathcal{K}^{2}{ }_{2}+\mathcal{K}^{1}{ }_{1} \mathcal{K}^{2}{ }_{2}\right)\right. \\
& \left.+\alpha\left[\mathcal{K}_{0}^{0}{ }_{0} \mathcal{K}^{1}{ }_{1}+\mathcal{K}^{2}{ }_{2}\left(2 \mathcal{K}^{0}{ }_{0}+2 \mathcal{K}^{1}{ }_{1}+\mathcal{K}^{2}{ }_{2}\right)\right]+\mathcal{K}^{0}{ }_{0}+\mathcal{K}^{1}{ }_{1}+2 \mathcal{K}^{2}{ }_{2}\right\} g_{22} \\
\mathcal{H}_{44}^{(5)} & =\mathcal{H}_{33}^{(5)}=\mathcal{H}_{22}^{(5)}
\end{aligned}
$$

along with that of $s_{\mu \nu}^{(5)}(f)$ defined as

$$
\begin{aligned}
& s_{00}^{(5)}=\left(1-\mathcal{K}_{0}^{0}\right)\left[\alpha_{5} \mathcal{K}^{1}{ }_{1}\left(\mathcal{K}^{2}{ }_{2}\right)^{3}+\alpha_{4}\left(\mathcal{K}^{2}{ }_{2}\right)^{2}\left(3 \mathcal{K}^{1}{ }_{1}+\mathcal{K}^{2}{ }_{2}\right)+3 \alpha_{3} \mathcal{K}^{2}{ }_{2}\left(\mathcal{K}^{1}{ }_{1}+\mathcal{K}^{2}{ }_{2}\right)\right. \\
& \left.+\mathcal{K}^{1}{ }_{1}+3 \mathcal{K}_{2}^{2}\right] f_{00} \\
& s_{11}^{(5)}=\left(1-\mathcal{K}^{1}{ }_{1}\right)\left[\alpha_{5} \mathcal{K}^{0}{ }_{0}\left(\mathcal{K}^{2}{ }_{2}\right)^{3}+\alpha_{4}\left(\mathcal{K}^{2}{ }_{2}\right)^{2}\left(3 \mathcal{K}^{0}{ }_{0}+\mathcal{K}^{2}{ }_{2}\right)+3 \alpha_{3} \mathcal{K}^{2}{ }_{2}\left(\mathcal{K}^{0}{ }_{0}+\mathcal{K}^{2}{ }_{2}\right)\right. \\
& \left.+\mathcal{K}_{0}^{0}+3 \mathcal{K}_{2}^{2}\right] f_{11} \text {, } \\
& s_{22}^{(5)}=\left(1-\mathcal{K}^{2}{ }_{2}\right)\left\{\alpha_{5} \mathcal{K}^{0}{ }_{0} \mathcal{K}^{1}{ }_{1}\left(\mathcal{K}^{2}{ }_{2}\right)^{2}+\alpha_{4} \mathcal{K}^{2}{ }_{2}\left(2 \mathcal{K}^{0}{ }_{0} \mathcal{K}^{1}{ }_{1}+\mathcal{K}^{0}{ }_{0} \mathcal{K}^{2}{ }_{2}+\mathcal{K}^{1}{ }_{1} \mathcal{K}^{2}{ }_{2}\right)\right. \\
& \left.+\alpha_{3}\left[\left(\mathcal{K}^{2}{ }_{2}\right)^{2}+2 \mathcal{K}^{2}{ }_{2}\left(\mathcal{K}_{0}^{0}+\mathcal{K}^{1}{ }_{1}\right)+\mathcal{K}^{0}{ }_{0} \mathcal{K}^{1}{ }_{1}\right]+\mathcal{K}^{0}{ }_{0}+\mathcal{K}^{1}{ }_{1}+2 \mathcal{K}^{2}{ }_{2}\right\} f_{22}, \\
& s_{44}^{(5)}=s_{33}^{(5)}=s_{22}^{(5)} \text {. }
\end{aligned}
$$


These Bianchi constraint equations look very complicated to solve. Fortunately, a simple solution to them can be found to be

$$
f_{\mu \nu}=(1-\bar{C})^{2} g_{\mu \nu},
$$

which corresponds to $\mathcal{K}^{2}{ }_{2}=\mathcal{K}^{1}{ }_{1}=\mathcal{K}^{0}{ }_{0}=\bar{C}$, where $\bar{C}$ is a constant. It is noted that this solution has been discussed extensively for a number black hole solutions of the 4D massive bi-gravity, e.g., see Ref. [12]. Consequently, the massive gravitons of the 5D massive bigravity turn out to be an effective cosmological constant. In particular, the corresponding effective cosmological constant for the physical and reference metrics are defined to be

$$
\bar{\Lambda}_{0}^{g}=-\frac{\bar{C}}{\tilde{M}_{g}^{2}}\left(\sigma \bar{C}^{3}+4 \beta \bar{C}^{2}+6 \alpha \bar{C}+4\right) ; \bar{\Lambda}_{0}^{f}=\frac{\bar{C}}{\tilde{M}_{f}^{2}(1-\bar{C})^{4}}\left(\alpha_{5} \bar{C}^{3}+4 \alpha_{4} \bar{C}^{2}+6 \alpha_{3} \bar{C}+4\right),
$$

respectively. As a result, the value of the constant $\bar{C}$ can be found from the equation $\bar{\Lambda}_{0}^{g}=$ $(1-\bar{C})^{2} \bar{\Lambda}_{0}^{f}$, which can be expanded to be

$$
\begin{aligned}
& \sigma \bar{C}^{5}-2(\sigma-2 \beta) \bar{C}^{4}+\left(\sigma-8 \beta+6 \alpha+\alpha_{5} \tilde{M}^{2}\right) \bar{C}^{3}+4\left(\beta-3 \alpha+\alpha_{4} \tilde{M}^{2}+1\right) \bar{C}^{2} \\
& +2\left(3 \alpha+3 \alpha_{3} \tilde{M}^{2}-4\right) \bar{C}+4\left(\tilde{M}^{2}+1\right)=0 .
\end{aligned}
$$

Here $\tilde{M}_{g}^{2} \equiv M_{g}^{2} /\left(m^{2} M_{\text {eff }}^{2}\right), \tilde{M}_{f}^{2} \equiv M_{f}^{2} /\left(m^{2} M_{\text {eff }}^{2}\right)$, and $\tilde{M}^{2} \equiv \tilde{M}_{g}^{2} / \tilde{M}_{f}^{2}$ being additional parameters. Hence, the Einstein field equations (43) and (44) for the physical and reference sectors become

$$
R_{\mu v}(g)-\frac{1}{2} g_{\mu v} R(g)+\bar{\Lambda}_{0}^{g} g_{\mu \nu}=0 ; R_{\mu v}(f)-\frac{1}{2} f_{\mu v} R(f)+\bar{\Lambda}_{0}^{f} f_{\mu v}=0,
$$

respectively. As a result, Eq. (64) of the physical metric does admit the SchwarzschildTangherlini-(anti)-de Sitter black holes as its solution, whose metric is given by [17, 19]

$$
d s^{2}=-f(r) d t^{2}+\frac{d r^{2}}{f(r)}+r^{2} d \Omega_{3}^{2} ; N_{1}^{2}(r)=F_{1}^{2}(r)=f(r)=1-\frac{\mu}{r^{2}}-\frac{\bar{\Lambda}_{0}^{g}}{6} r^{2} ; H_{1}^{2}(r)=1 .
$$

\section{Conclusions}

We have shown that the Cayley-Hamilton theorem is the effective method to construct any $n$-dimensional $(n>4)$ graviton terms, which vanish identically in any 4D spacetimes but will not disappear in $m$-dimensional $(m \geq n)$ ones. Then, we have proposed to study the 5D nonlinear massive gravity as well as massive bi-gravity with a full set of graviton terms, i.e., $\mathcal{L}_{i}(i=2-5)[16,17]$. In particular, we have pointed out that the Schwarzschild-Tangherlini(anti)-de Sitter black holes do exist in not only the 5D nonlinear massive gravity but also the 5D massive bi-gravity thanks to the constant-like behavior of the massive graviton terms. We hope that our research could shed more light on higher dimensional massive (bi-)gravity.

\section{Acknowledgments}

The author would like to thank the organizers of XLVIII International Symposium on Multiparticle Dynamics (ISMD2018), especially Professors Kok Khoo Phua, Nguyen Anh Ky, and Aik Hui (Phil) Chan, very much for their supports and warm hospitality. The author would also like to thank the Institute of Advanced Studies, Nanyang Technological University as well as the National University of Singapore very much for their supports and warm hospitality. This work is supported by the Vietnam National Foundation for Science and Technology Development (NAFOSTED) under Grant No. 103.01-2017.12. 


\section{References}

[1] M. Fierz and W. Pauli, Proc. R. Soc. Lond. A 173, 211 (1939).

[2] H. van Dam and M. J. G. Veltman, Nucl. Phys. B 22, 397 (1970).

[3] V. I. Zakharov, JETP Lett. 12, 312 (1970).

[4] A. I. Vainshtein, Phys. Lett. B 39, 393 (1972).

[5] D. G. Boulware and S. Deser, Phys. Rev. D 6, 3368 (1972).

[6] N. Arkani-Hamed, H. Georgi, and M. D. Schwartz, Ann. Phys. (N.Y.) 305, 96 (2003); S. L. Dubovsky, J. High Energy Phys. 10, 076 (2004); P. Creminelli, A. Nicolis, M. Papucci, and E. Trincherini, J. High Energy Phys. 09, 003 (2005); K. Hinterbichler, Rev. Mod. Phys. 84, 671 (2012).

[7] C. de Rham, G. Gabadadze, and A. J. Tolley, Phys. Rev. Lett. 106, 231101 (2011); C. de Rham and G. Gabadadze, Phys. Rev. D 82, 044020 (2010).

[8] S. F. Hassan and R. A. Rosen, Phys. Rev. Lett. 108, 041101 (2012); S. F. Hassan and R. A. Rosen, J. High Energy Phys. 04, 123 (2012); S. F. Hassan, R. A. Rosen, and A. Schmidt-May, J. High Energy Phys. 02, 026 (2012); S. F. Hassan, A. Schmidt-May, and M. von Strauss, Phys. Lett. B 715, 335 (2012); J. Kluson, Phys. Rev. D 86, 124005 (2012); J. Kluson, Phys. Rev. D 86, 044024 (2012); C. de Rham, G. Gabadadze, and A. J. Tolley, J. High Energy Phys. 11, 093 (2011); C. de Rham, G. Gabadadze, and A. J. Tolley, Phys. Lett. B 711, 190 (2012); M. Mirbabayi, Phys. Rev. D 86, 084006 (2012); A. Golovnev, Phys. Lett. B 707, 404 (2012); T. Kugo and N. Ohta, Prog. Theor. Exp. Phys. 043B04 (2014).

[9] C. de Rham, Living Rev. Relativity 17, 7 (2014).

[10] K. Koyama, G. Niz, and G. Tasinato, Phys. Rev. Lett. 107, 131101 (2011); T. M. Nieuwenhuizen, Phys. Rev. D 84, 024038 (2011); L. Berezhiani, G. Chkareuli, C. de Rham, G. Gabadadze, and A. J. Tolley, Phys. Rev. D 85, 044024 (2012); Y. F. Cai, D. A. Easson, C. Gao, and E. N. Saridakis, Phys. Rev. D 87, 064001 (2013); E. Babichev and A. Fabbri, Phys. Rev. D 90, 084019 (2014).

[11] S. F. Hassan and R. A. Rosen J. High Energy Phys. 02, 126 (2012); S. F. Hassan and R. A. Rosen, J. High Energy Phys. 07, 009 (2011).

[12] A. Schmidt-May and M. von Strauss, J. Phys. A 49, 183001 (2016).

[13] S. F. Hassan, A. Schmidt-May, and M. von Strauss, Classical Quantum Gravity 30, 184010 (2013).

[14] K. Hinterbichler and R. A. Rosen, J. High Energy Phys. 07, 047 (2012); M. F. Paulos and A. J. Tolley, J. High Energy Phys. 09, 002 (2012); Q. G. Huang, K. C. Zhang, and S. Y. Zhou, J. Cosmol. Astropart. Phys. 08, 050 (2013).

[15] R. G. Cai, Y. P. Hu, Q. Y. Pan, and Y. L. Zhang, Phys. Rev. D 91, 024032 (2015); J. Xu, L. M. Cao, and Y. P. Hu, Phys. Rev. D 91, 124033 (2015); Z. Zhou, J. P. Wu, and Y. Ling, J. High Energy Phys. 08, 067 (2015).

[16] T. Q. Do, Phys. Rev. D 93, 104003 (2016).

[17] T. Q. Do, Phys. Rev. D 94, 104022 (2016).

[18] S. Lipschutz and M. L. Lipson, Schaum's Outline of Linear Algebra (New York: McGraw-Hill, 2009), pp. 294.

[19] F. R. Tangherlini, Nuovo Cim. 27, 636 (1963).

[20] T. Q. Do and W. F. Kao, Phys. Rev. D 88, 063006 (2013). 